\title{
The correlation between serum vascular endothelial growth factor (VEGF) and tumor VEGF receptor 3 in colorectal cancer
}

\author{
André Goulart ${ }^{1,2}$, Carla Ferreira ${ }^{2}$, Ana Rodrigues ${ }^{2}$, Barbara Coimbra², Nuno Sousa ${ }^{2}$, Pedro Leão ${ }^{1,2}$ \\ ${ }^{1}$ General Surgery, Hospital de Braga, Braga, Portugal \\ ${ }^{2}$ Life and Health Science Research Institute (ICVS), School of Medicine, University of Minho, Braga, Portugal
}

\begin{abstract}
Purpose: Despite plasma biomarkers offering a number of advantages over tissue-based markers, the relationship between serum vascular endothelial growth factor (VEGF) and VEGF receptor (VEGF-R) tumor expression in colorectal cancer (CRC) is still unclear. This study was designed to establish the relationship between the concentration of serum VEGF and tumor VEGF-R expression in patients with CRC.

Methods: A prospective study of consecutive patients undergoing elective colorectal surgery during 1 year. Preoperative VEGF was determined by enzyme-linked immunosorbent assay and VEGF-R3 by immunochemistry.

Results: The initial sample included 134 patients with CRC diagnosis. Results showed significant association of serum values of VEGF with VEGF-R3 expression ( $P<0.001$ ), even in the presence of confounders (sex, age, body mass index, tumor location, and surgical approach). The estimated effect size was high $\left(\eta^{2}=0.35\right)$.

Conclusion: Serum VEGF has a significant correlation with tumoral VEGF-R3 expression in CRC.

[Ann Surg Treat Res 2019;97(1):15-20]
\end{abstract}

Key Words: Colorectal neoplasms, Vascular endothelial growth factors, Enzyme-linked immunosorbent assay, Immunohistochemistry

\section{INTRODUCTION}

Vascular endothelial growth factor (VEGF) is the most widely studied angiogenic factor, being considered crucial for tumor angiogenesis [1]. VEGF presents itself as a signal protein that stimulates the growth of new blood vessels and it is thought to facilitate the metastatic spread of tumor cells [1,2]. The pathway for signal transduction of VEGF is composed of 5 glycoproteins belonging to the VEGF family including VEGF-A, VEGF-B, VEGF-C, VEGF-D, and placental growth factor, 3 receptors; VEGF receptor-1 (FTL-1), VEGF-R2 (FLK-1/KDR), and VEGF-R3 (FLT4), and 2 coreceptors (NRP-1, NRP-2) [2]. The three VEGF-Rs participate differently in angiogenesis and lymphangiogenenis:
VEGF-R1 and VEGF-R2 mediate angiogenesis, and VEGF-R3 is mostly involved in lymphangiogenesis [2]. The main inducers of new lymphatic vessels are the principal ligands of VEGF-R3 [2]. In addition to the role in tumor growth, it appears that VEGF also has autocrine functions, acting as a survival factor for tumor cells, protecting them from stresses such as hypoxia, chemotherapy (CT) and radiotherapy (RT) [3]. Exogenous VEGF could attenuate the effect of RT, so combining RT with VEGF inhibitor could be more effective than RT alone [4,5]. A recent review that explores the benefit of adding target therapy to neoadjuvant chemotherapy in locally advanced rectal cancer leaves several open questions in conclusion, such as that patient selection should be based on potential predictive biomarkers
Received October 18, 2018, Revised December 13, 2018, Accepted December 21, 2018

Corresponding Author: André Goulart

General Surgery, Hospital de Braga, 4701-965 Braga, Portugal

Tel: +351-969020736

E-mail: andre.b.goulart@gmail.com

ORCID code: https://orcid.org/0000-0002-9167-3256
Copyright (C) 2019, the Korean Surgical Society

(c) Annals of Surgical Treatment and Research is an Open Access Journal. All articles are distributed under the terms of the Creative Commons Attribution NonCommercial License (http://creativecommons.org/licenses/by-nc/4.0/) which permits unrestricted non-commercial use, distribution, and reproduction in any medium, provided the original work is properly cited. 
of response, such as free VEGF, in order to define a subgroup of patients who are more likely to benefit from this form of therapy [6].

VEGF can be measured in the tumor tissue by immunohistochemistry or reverse transcription polymerase chain reaction or in the plasma by enzyme-linked immunosorbent assay (ELISA) method [7]. Detection of serum VEGF has been investigated as a potential serum diagnostic marker for malignant disease, and was found to increase concentrations of free VEGF in various types of cancer, including those of gastrointestinal etiology [8,9]. However, the relationship between the pattern of production of VEGF protein in tumor tissues and its concentration in the circulation remains unclear $[10,11]$.

Plasma biomarkers offer a number of advantages over tissuebased markers. The potential of serum concentration of VEGF being representative of tumor VEGF opens new pathways for further investigation, preoperative prognostic information and treatment response. The absence of papers designed to verify this relationship lead us to design a study with the intention of clarifying the relationship between the concentration of serum VEGF and tumor VEGF-R expression in patients with CRC.

\section{METHODS}

\section{Study oversight and patient inclusion}

Between August 2015 and August 2016 all patients with a confirmed diagnosis of colorectal adenocarcinoma who underwent elective surgery at our institution were enrolled. Patients who presented evidence of metastasis before or at surgery, necessity of removal of other organs due to tumor invasion, synchronous tumors or history of other malignant tumors within 5 years, history of familial adenomatous polyposis and hereditary nonpolyposis colorectal carcinoma were excluded. We also excluded patients who did not do preoperative assessment, did not have preoperative blood samples collected, malignant polyp excised but without tumor in the colectomy specimen, pathologic completed response after neoadjuvant $\mathrm{CT}$ - ypT0, or adenocarcinoma in situ and mucinous adenocarcinoma.

Written informed consent was obtained from all participant patients. This study was approved by the Ethics Committee for Health of Hospital de Braga (authorization number 60/2017).

\section{Data collection}

At 1 day before surgery, the same nurse performed an anthropometric evaluation of the patients that included body weight and height and obtained a sample for the evaluation of serum VEGF. A biopsy representative of each tumor was taken from Department of Pathology.

\section{Immunohistochemistry}

One representative histological specimen of each case at the deepest invaded area of the CRC lesion was selected by same pathologist for immunohistochemistry. Immunohistochemical staining was performed on the samples with a thickness of $2.5 \mu \mathrm{m}$, which were cut using the Thermo - MicroM HM355S with a simultaneous water bath at $56^{\circ} \mathrm{C}$ for flattening out and drying tissue sections (Medite TFB45). After the water bath, the cut samples were placed on specific slides for a period of 20 minutes at $60^{\circ} \mathrm{C}$ in the Memmert Model 100-800. For the removal of paraffin, BondTM Dewax Solution (Catalog number AR9222, Leica Biosystems, Wetzlar, Germany) was used followed by VEGF-R protocol for Mouse Monoclonal Antibody VEGFR-3 (dilution 1:50; clone KLT9; Product code NCL-L-VEGFR-3, Leica Biosystems). The antibody was diluted with Novocastra TM IHC Diluent (Product Code RE7133, Leica Biosystems). All sections were incubated with primary antibody incubation for 60 minutes at $25^{\circ} \mathrm{C}$. Staining was performed using the BOND - MAX Automated from Leica following the manufacturer's procedures. It was used with the following products: BondTM Wash Solution 10X Concentrate (Catolog number AR9590, Leica Biosystems), BondTM Epitope Retrival solution 1 (Catolog number AR 9961, Leica Biosystems) and BondTM Polymer Refine Detection (Catolog number DS9800, Leica Biosystems).

Then the slides were washed in distilled water. Afterwards, the slides were dehydrated in an ascending series of alcohols (70\%, 96\%, and 100\%) and made diaphanous with xylene, and finally mounted with Entellan glue.

\section{Microscopic assessment of VEGF-R3 expression}

VEGF-R3 staining was graded according to the intensity and extent of staining of the endothelium of the vessels as previously published [12]. The scale presenting hence is the following: $0=$ absent (Fig. 1A), $1=$ weak/very limited moderate staining (Fig. 1B), 2 = moderate widespread/strong localized staining (Fig. 1C) and $3=$ strong widespread (Fig. 1D). This was assessed under $\times 100$ magnification for all of the sections taken.

\section{Serum VEGF determination}

For determination of serum VEGF levels, blood samples were collected from the day before surgery. Serum samples were obtained by centrifugation at 3,000 revolutions per minute for 10 minutes and were stored at $-80^{\circ} \mathrm{C}$ until use. Serum levels of VEGF were determined using a commercially available sandwich enzyme immunoassay kit (Human VEGF ELISA kit; Catalog number KHG0111, KHG0112, Life Technologies, Carlsbad, CA, USA). Samples were prepared and tested in duplicate according to the manufacturer's instructions. The reported detection limit is $<5 \mathrm{pg} / \mathrm{mL}$. 

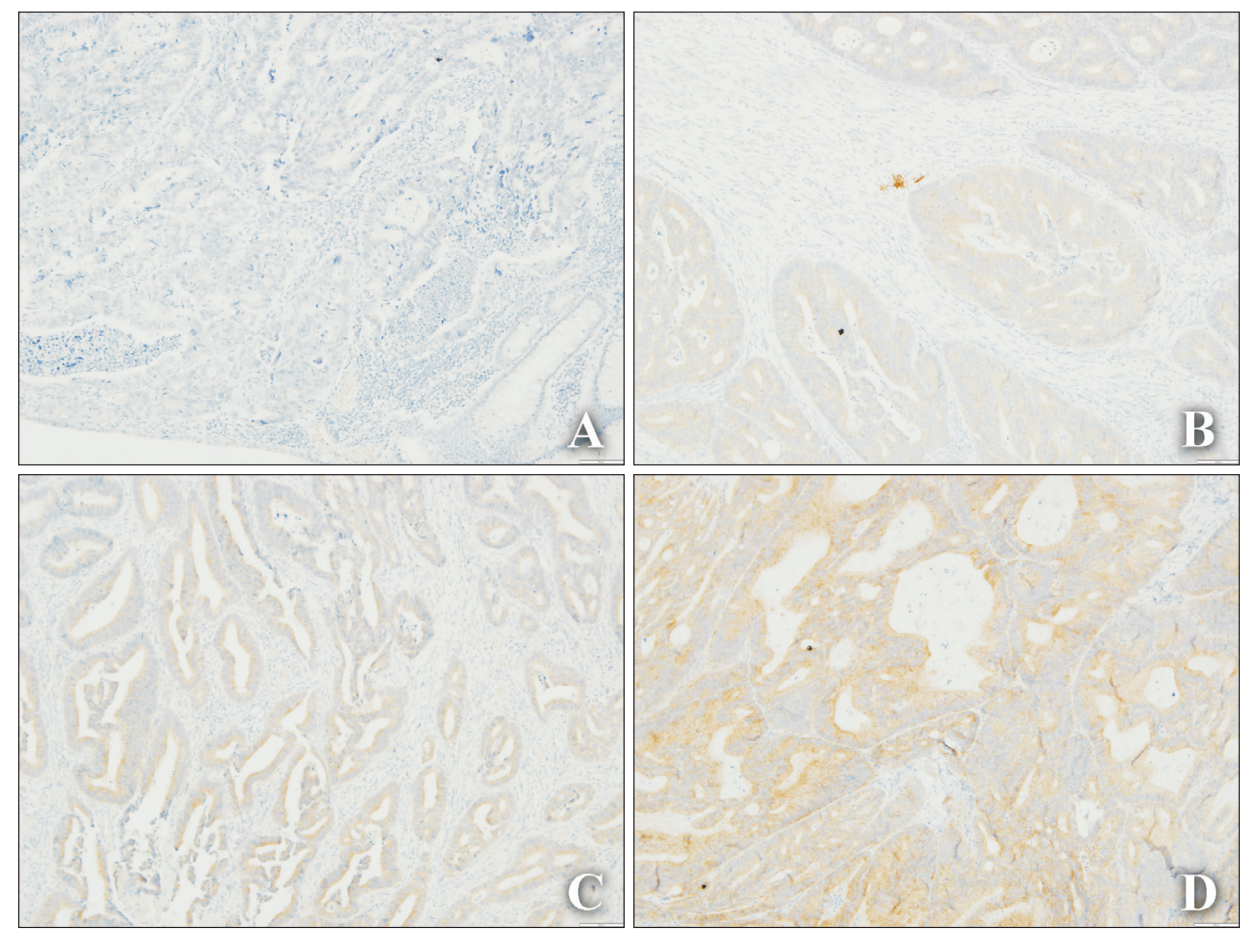

Fig. 1. Immunohistochemical staining for VEGF-R3 in malignant colorectal endothelium. (A) Absent expression of VEGF-R3 in CRC $(\times 100)$. (B) Weak/very limited moderate staining of VEGF-R3 (×100). (C) Moderate widespread/strong localized staining of VEGF-R3 (×100). (D) Strong widespread staining of VEGF-R3 (×100). VEGF, vascular endothelial growth factor; VEGFR3, VEGF receptor 3; CRC, colorectal cancer.

\section{Statistical analysis}

Statistical analysis was performed using IBM SPSS Statistics ver. 24.0 (IBM Co., Armonk, NY, USA). Descriptive statistics were presented as means (M) and standard deviations (SD) for quantitative variables; categorical variables we computed frequencies $(\mathrm{n})$ and percentages (\%). Normality was checked with Shapiro-Wilks test assuming normality for $\mathrm{P}>0.05$. Levene test was used for assessing the homogeneity of variances, making this assumption when $\mathrm{P}>0.05$. One-way analyses of variance (ANOVA) was used to compare serum values of VEGF. Spearman correlation was used to measure the association between these variables. Confounders were controlled by conducting an analyses of covariance (ANCOVA). Effect size was measured with eta ${ }^{2}\left(\eta^{2}\right)$, considering low effect $\left(\eta^{2}\right.$ $=0.01)$, moderate effect $\left(\eta^{2}=0.06\right)$ and high effect $\left.\eta^{2}=0.14\right)$. Significant results were considered for $\mathrm{P}<0.05$.

\section{RESULTS}

\section{Study population and baseline characteristics}

The initial sample included 134 patients with CRC diagnoses. From these, 60 patients (44.8\%) were excluded for the following reasons: no preoperative assessment $(26 ; 19.4 \%)$, lack of preoperative blood samples (19; 14.2\%), pathologic completed response after neoadjuvant $\mathrm{CT}$ - ypT0 $(5 ; 3.7 \%)$, malignant polyp excised but without tumor in the colectomy specimen $(4 ; 3.0 \%)$, adenocarcinoma in situ $(4 ; 3.0 \%)$, mucinous adenocarcinoma (1; $0.7 \%$ ), and lack of sample $(1 ; 0.7 \%)$. The final sample was composed of a total of 74 patients with CRC diagnoses, 47
Table 1. Patient and tumor characteristics

\begin{tabular}{lc}
\hline \multicolumn{1}{c}{ Characteristic } & Value \\
\hline Age $(y r)$ & $68.34 \pm 12.69$ \\
Sex & $47(63.5)$ \\
Male & $27(36.5)$ \\
Female & \\
Location & $18(24.3)$ \\
Rectum & $56(75.7)$ \\
Colon & \\
Surgical approach & $38(51.4)$ \\
Laparotomy & $33(44.6)$ \\
Laparoscopy & $3(4.1)$ \\
Conversion from laparoscopy & $26.97 \pm 4.08$ \\
Body mass index (kg/m $\left.{ }^{2}\right)$ & \\
T classification (TNM) & $5(6.8)$ \\
T1 & $21(28.4)$ \\
T2 & $44(59.5)$ \\
T3 & $4(5.4)$ \\
T4 & \\
N classification (TNM) & $51(68.9)$ \\
N0 & $23(31.1)$ \\
N+ & \\
Dukes stage & $20(27.0)$ \\
Dukes A & $31(41.9)$ \\
Dukes B & $23(31.1)$ \\
Dukes C & \\
\hline
\end{tabular}

Values are presented as mean \pm standard deviation or number (\%). 
Table 2. ANOVA comparison for serum VEGF and tumoral VEGF-R3 expression

\begin{tabular}{lcccc}
\hline & $\begin{array}{c}\text { Absent } \\
(\mathrm{n}=6)\end{array}$ & $\begin{array}{c}\text { Weak/very limited } \\
\text { moderated } \\
(\mathrm{n}=49)\end{array}$ & $\begin{array}{c}\text { Moderated } \\
\text { widespread/strong } \\
\text { localized }(\mathrm{n}=13)\end{array}$ & $\begin{array}{c}\text { Strong widespread } \\
(\mathrm{n}=6)\end{array}$ \\
Serum VEGF concentration & $36.29 \pm 12.55$ & $49.68 \pm 18.96$ & $65.35 \pm 36.21$ & $\begin{array}{c}126.39 \pm 77.31 \\
\text { ANOVA }\end{array}$ \\
\hline
\end{tabular}

Values are presented as mean \pm standard deviation.

ANOVA, analysis of variance; VEGF, vascular endothelial growth factor; VEGF-R3, VEGF receptor 3.

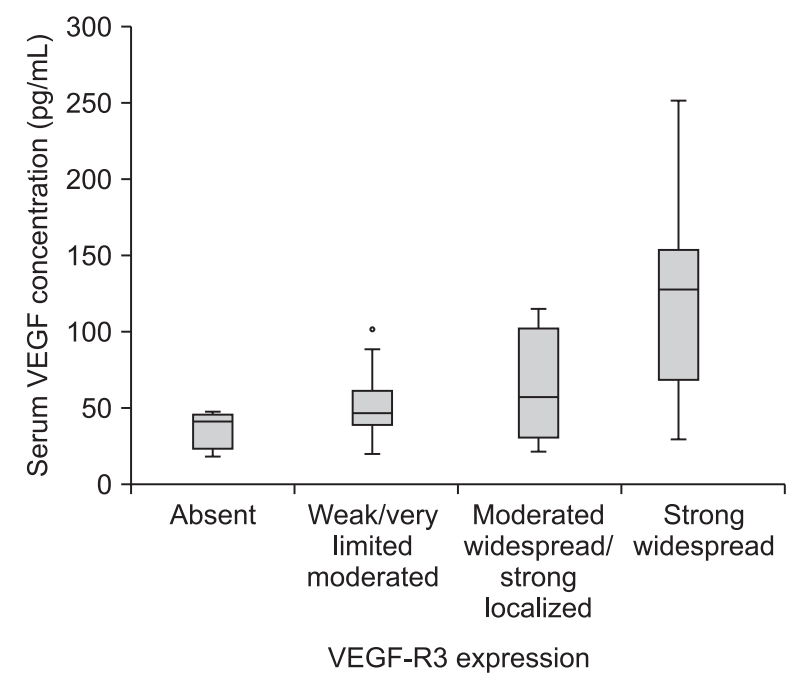

Fig. 2. Serum values of VEGF distribution by immunochemistry VEGF-R3 expression. VEGF, vascular endothelial growth factor; VEGF-R3, VEGF receptor 3; CRC, colorectal cancer.

males (63.5\%) and 27 females (36.5\%) with a mean age of 68 years. Other patient and pathologic characteristics are described in Table 1.

\section{Analytic results}

After checking for normality and variances homogeneity, ANOVA results showed overall significant differences $(\mathrm{P}<0.01$, $\eta^{2}=0.34$ ). Tukey multiple comparisons test showed significant differences regarding the comparison of strong widespread staining (mean \pm SD, $126.39 \pm 77.31$ ) with all other categories: moderated widespread/strong localized (mean \pm SD, $65.35 \pm$ 36.21), weak/very limited moderated (mean \pm SD, $49.68 \pm$ 18.96), and absent (mean \pm SD, $36.29 \pm 12.55$ ) (Table 2, Fig. 2). Spearman correlation showed significant association between these variables $(\rho=0.348, P=0.002$ ).

An ANCOVA analysis intending to control for the confounders: age, sex, body mass index, tumor location, surgical approach, TNM, and Dukes classification was performed. Results showed significant association of serum values of VEGF with VEGF-R3 expression $(P<0.01)$, even in the presence of all referred confounders. Effect size estimate was $\eta^{2}=0.35$ (Table 3).
Table 3. ANCOVA analysis of covariance for serum values of VEGF by VEGF-R3 expression adjusted for age, sex, body mass index, location, surgical approach, T classification, $N$ classification, and Dukes classification

\begin{tabular}{lrc}
\hline \multicolumn{1}{c}{ Variable } & P-value & $\eta^{2}$ \\
VEGF-R3 expression & $<0.001$ & 0.35 \\
Age & 0.315 & 0.02 \\
Sex & 0.197 & 0.03 \\
Body mass index & 0.280 & 0.02 \\
Location & 0.747 & 0.02 \\
Surgical approach & 0.809 & 0.01 \\
T classification (TNM) & 0.218 & 0.02 \\
N classification (TNM) & 0.260 & 0.02 \\
Dukes classification & 0.185 & 0.03 \\
\hline
\end{tabular}

ANCOVA, analysis of covariance; VEGF, vascular endothelial growth factor; VEGF-R3, VEGF receptor 3.

\section{DISCUSSION}

The relationship between VEFG and CRC outcomes is controversial. Some studies showed that VEGF has no significant prognostic value in CRC [13], but many others have demonstrated an association between overexpression of VEGF and poor CRC outcomes: the overexpression of VEGF and VEGF-R in CRC tissue indicates poor prognosis $[4,14,15]$, predicts early relapse [16], and preoperative VEGF serum concentration predicts poor disease-specific survival and disease-free survival in colon cancer patients [17]. When comparing patients who had metastatic tumors compared with patients who had nonmetastatic tumors, it was found that VEGF expression was higher in the first group [18-20]. Takahashi et al. [21] demonstrated that VEGF expression levels in patients with lymph node negative CRC was significantly associated with time to recurrence, and Cascinu et al. [22] verified the association between positive VEGF tumor status with a significant reduction in the 5-year disease-free survival rate. Some studies have shown that VEGF is also a useful prognostic marker, by significantly correlating with angio-lymphatic invasion, lymph node status, and depth of invasion, although it is not an independent prognostic factor [10].

Regardless of serum VEGF collected before the surgery theoretically presenting many advantages over tumoral VEGF, 
only a few studies evaluated the prognostic impact of VEGF serum levels in patients with CRC. The Danish Colorectal Cancer Study Group conducted a large study which suggested a biologically relevant role for serum VEGF concentrations in patients with $\mathrm{CRC}$, after having found that high preoperative VEGF concentrations were associated with a reduced overall survival [23-25]. In the same way, Nakayama et al. [26] reported on elevated circulating levels of VEGF in patients with CRC who had more advanced disease and in patients who experienced tumor recurrence.

Probably one of the reasons for serum VEGF being used as prognostic marker in only a few papers is related to the absence of studies designed with the purpose of clarifying the relationship between serum and tumoral VEGF. Our work showed a strong association $\left(P<0.001, \eta^{2}=0.34\right)$ between preoperative serum VEGF and VEGF-R3 tumor expression even after controlling for potential confounders. This association could be explained by previous studies that show that VEGF is expressed in a wide variety of human tissues, particularly high quantity in tumors, and that, in vitro, many tumor cell lines secrete VEGF [17]. Thus, it seems logical that patients with tumor cells with a higher expression of VEGF-R present higher levels of serum VEGF in comparison with tumors with lesser VEGF-R expression.

These results open up new horizons in terms of investigation as a potential biomarker in selection of treatment and prognostic information. In the near future, it may be possible to implement a patient selection strategy to effectively identify those patients who are most likely to benefit from neoadjuvant treatment with VEGF inhibitors based on this confirmed relationship between serum and tumoral VEGF expression. However, due to limitation of sample size, our work needs to be considered as a pilot study. Further studies with a larger sample need to be developed in order to confirm these results. Moreover, our study only focuses on the relationship between serum VEGF and tumor VEGF-R expression, so the prognostic significance of serum VEGF still requires further investigation.

\section{CONFLICTS OF INTEREST}

No potential conflict of interest relevant to this article was reported.

\section{REFERENCES}

1. Larijani LV, Ghasemi M, Charati JY, Mehrabian-Fard M, Saravi NS. Evaluation of VEGF immunohistochemical expression and correlation with clinicopathologic features in colorectal cancer. Govaresh 2015;20:199-204.

2. Al-Rawi MA, Mansel RE, Jiang WG. Molecular and cellular mechanisms of lymphangiogenesis. Eur J Surg Oncol 2005;31:117-21.

3. Jurgensmeier JM, Schmoll HJ, Robertson JD, Brooks L, Taboada M, Morgan SR, et al. Prognostic and predictive value of VEGF, sVEGFR-2 and CEA in mCRC studies comparing cediranib, bevacizumab and chemotherapy. Br J Cancer 2013;108:131623.

4. Peng Y, Wang L, Du C, Gu J. Expression of vascular endothelial growth factor can predict distant metastasis and diseasefree survival for clinical stage III rectal cancer following 30-Gy/10-f preoperative radiotherapy. Int J Colorectal Dis 2012;27: 1555-60.
5. Gupta VK, Jaskowiak NT, Beckett MA, Mauceri HJ, Grunstein J, Johnson RS, et al. Vascular endothelial growth factor enhances endothelial cell survival and tumor radioresistance. Cancer J 2002;8:4754.

6. Benevento I, De Felice F, Musio D, Tombolini $\mathrm{V}$. The addition of target therapy to neoadjuvant chemoradiotherapy in locally advanced rectal cancer: a review. Chemotherapy 2017;62:314-22.

7. Byrne AM, Bouchier-Hayes DJ, Harmey JH. Angiogenic and cell survival functions of vascular endothelial growth factor (VEGF). J Cell Mol Med 2005:9:777-94.

8. Bottomley MJ, Webb NJ, Watson CJ, Holt L, Bukhari M, Denton J, et al. Placenta growth factor (PlGF) induces vascular endothelial growth factor (VEGF) secretion from mononuclear cells and is co-expressed with VEGF in synovial fluid. Clin Exp Immunol 2000;119:182-8.

9. Jelkmann W. Pitfalls in the measurement of circulating vascular endothelial growth factor. Clin Chem 2001:47:617-23.

10. Martins SF, Reis RM, Rodrigues AM, Baltazar F, Filho AL. Role of endoglin and VEGF family expression in colorectal cancer prognosis and anti-angiogenic therapies. World J Clin Oncol 2011;2:27280.

11. Cressey R, Wattananupong $\mathrm{O}$, Lertprasertsuke N, Vinitketkumnuen U. Alteration of protein expression pattern of vascular endothelial growth factor (VEGF) from soluble to cell-associated isoform during tumourigenesis. BMC Cancer 2005;5:128.

12. White JD, Hewett PW, Kosuge D, McCulloch T, Enholm BC, Carmichael J, et al. Vascular endothelial growth factor-D expression is an independent prognostic marker for survival in colorectal carcinoma. Cancer Res 2002;62:1669-75.

13. Zheng S, Han MY, Xiao ZX, Peng JP, Dong Q. Clinical significance of vascular endothelial growth factor expression and neovascularization in colorectal 
carcinoma. World J Gastroenterol 2003;9: 1227-30.

14. Martins SF, Garcia EA, Luz MA, Pardal F, Rodrigues M, Filho AL. Clinicopathological correlation and prognostic significance of VEGF-A, VEGF-C, VEGFR-2 and VEGFR-3 expression in colorectal cancer. Cancer Genomics Proteomics 2013;10:5567.

15. Des Guetz G, Uzzan B, Nicolas P, Cucherat M, Morere JF, Benamouzig R, et al. Microvessel density and VEGF expression are prognostic factors in colorectal cancer. Meta-analysis of the literature. Br J Cancer 2006;94:1823-32.

16. Tsai HL, Yang IP, Lin CH, Chai CY, Huang $\mathrm{YH}$, Chen $\mathrm{CF}$, et al. Predictive value of vascular endothelial growth factor overexpression in early relapse of colorectal cancer patients after curative resection. Int J Colorectal Dis 2013;28:41524.

17. De Vita F, Orditura M, Lieto E, Infusino S, Morgillo F, Martinelli E, et al. Elevated perioperative serum vascular endothelial growth factor levels in patients with colon carcinoma. Cancer 2004;100:270-8.

18. Takahashi Y, Kitadai Y, Bucana CD,
Cleary KR, Ellis LM. Expression of vascular endothelial growth factor and its receptor, KDR, correlates with vascularity, metastasis, and proliferation of human colon cancer. Cancer Res 1995:55:3964-8.

19. Tokunaga T, Oshika Y, Abe Y, Ozeki Y, Sadahiro S, Kijima H, et al. Vascular endothelial growth factor (VEGF) mRNA isoform expression pattern is correlated with liver metastasis and poor prognosis in colon cancer. Br J Cancer 1998;77:9981002.

20. Ishigami SI, Arii S, Furutani M, Niwano M, Harada T, Mizumoto M, et al. Predictive value of vascular endothelial growth factor (VEGF) in metastasis and prognosis of human colorectal cancer. Br J Cancer 1998;78:1379-84.

21. Takahashi Y, Tucker SL, Kitadai Y, Koura AN, Bucana CD, Cleary KR, et al. Vessel counts and expression of vascular endothelial growth factor as prognostic factors in node-negative colon cancer. Arch Surg 1997;132:541-6.

22. Cascinu S, Staccioli MP, Gasparini G, Giordani P, Catalano V, Ghiselli R, et al. Expression of vascular endothelial growth factor can predict event-free survival in stage II colon cancer. Clin Cancer Res 2000;6:2803-7.

23. Werther K, Christensen IJ, Brunner N, Nielsen HJ. Soluble vascular endothelial growth factor levels in patients with primary colorectal carcinoma. The Danish RANX05 Colorectal Cancer Study Group. Eur J Surg Oncol 2000;26:657-62.

24. Werther K, Christensen IJ, Nielsen HJ; Danish RANX05 Colorectal Cancer Study Group. The association between preoperative concentration of soluble vascular endothelial growth factor, perioperative blood transfusion, and survival in patients with primary colorectal cancer. Eur J Surg 2001;167:287-92.

25. Werther K, Christensen IJ, Nielsen HJ; Danish RANX05 Colorectal Cancer Study Group. Prognostic impact of matched preoperative plasma and serum VEGF in patients with primary colorectal carcinoma. Br J Cancer 2002;86:417-23.

26. Nakayama Y, Sako T, Shibao K, Okazaki K, Rempo N, Onitsuka K, et al. Prognostic value of plasma vascular endothelial growth factor in patients with colorectal cancer. Anticancer Res 2002;22:2437-42. 